\title{
Introduction of the second-generation direct-acting antivirals (DAAs) in chronic hepatitis C: a register-based study in Sweden
}

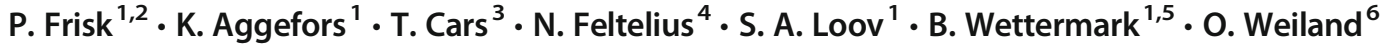

Received: 22 October 2017 / Accepted: 21 March 2018 / Published online: 9 April 2018

(C) The Author(s) 2018

\begin{abstract}
Purpose Introduction of the direct-acting antivirals (DAAs) for treatment of chronic hepatitis $\mathrm{C}$ (CHC) infection has been challenging in all health systems. In Sweden, a national protocol for managed introduction was developed. It was optional, but all county councils agreed to implement and follow it. The purpose of this study was to study (a) cure rates among all patients initiated on treatment in 2014-2015, (b) prescribers' adherence to the drug recommendations and treatment eligibility criteria in the protocol, and (c) introduction rate in the six Swedish healthcare regions.

Method A cross-sectional study where national data from the Prescribed Drug Register and the quality register InfCare Hepatitis defined the study population, and clinical data from the Patient Register and InfCare Hepatitis were used to monitor outcomes. Descriptive statistics were used.

Results A total of 3447 patients were initiated on treatment during 2014-2015. The overall cure rate, based on data from $85 \%$ of the cohort, was $96 \%$, with variation between genotypes. Adherence to drug recommendations increased over time and varied between 43.2 and $94.2 \%$. Adherence to the treatment eligibility criteria was initially $80 \%$ and increased to $87 \%$ when treatment restrictions were widened. The introduction rate differed initially between the regions and reached stable levels $15-18$ months after the launch of the first DAA.

Conclusion The estimated overall cure rate was $96 \%$, with some variations between genotypes. A high level of adherence to the introduction protocol as well as similar introduction rates in the health care regions indicate that the introduction protocol, alongside with other measures taken, contributed considerably to a rapid uptake and equal distribution of DAAs in Sweden.
\end{abstract}

Keywords Hepatitis C $\cdot$ Direct-acting antivirals $\cdot$ Effectiveness $\cdot$ Managed introduction

P. Frisk

pia.frisk@sll.se

1 Public Healthcare Services Committee, Stockholm County Council, P.O. Box 6909, S-102 39 Stockholm, Sweden

2 Department of Pharmaceutical Biosciences, Uppsala University, Uppsala, Sweden

3 Department of Medical Sciences, Uppsala University, Uppsala, Sweden

4 Medical Products Agency, Uppsala, Sweden

5 Department of Medicine Solna, Clinical Epidemiology Unit/Clin. Pharmacol, Karolinska Institutet, Stockholm, Sweden

6 Department of Medicine, Division of Infectious Diseases, Karolinska Institutet at Karolinska University Hospital Huddinge,

Stockholm, Sweden

\section{Introduction}

A majority of patients who become infected with hepatitis $\mathrm{C}$ virus ( $\mathrm{HCV}$ ) develop chronic hepatitis $\mathrm{C}(\mathrm{CHC})$. Left untreated, fibrosis in $\mathrm{CHC}$ will after on average 30 years have progressed to liver cirrhosis in a third of the patients, and many will develop hepatocellular cancer [1]. CHC is estimated to affect some 170 million people worldwide [1], with a rising incidence and prevalence [2]. Currently, the worldwide prevalence is estimated to approximately $2.8 \%$ [2]. Considering the consequences for both individuals and society, effective and affordable treatment options are urgently needed.

Drug treatment for chronic hepatitis $\mathrm{C}$ infection has improved markedly in recent years. The addition of firstgeneration protease inhibitors (PI), telaprevir or boceprevir, to pegIFN- $\alpha$ and ribavirin resulted in substantially increased cure rates for patients with HCV genotype 1 [3]. Addition of the first-generation PIs was however associated with many 
severe side effects and more complex drug-drug interactions than pegIFN and ribavirin treatment alone [4]. The adverse events associated with pegIFN and ribavirin therapies remained, and were enhanced with triple therapy including first-generation PIs. Furthermore, an additional pill-burden resulted in problems with poor adherence [4]. Clinical trial data with new direct-acting antiviral agents (DAAs) launched in 2014, however, showed high cure rates and few side effects with shorter treatment durations. Effective antiviral treatment for most genotypes had thus become available, and for the first time, IFN-free treatment options could be offered [5].

In Sweden, access to healthcare is provided by 21 county councils (autonomous healthcare regions), and is tax funded [6]. The right of licensed physicians to unrestricted prescribing and the fact that each county council is responsible by law for its own drug budget constitute the legal framework for prescribing drugs in Sweden. Prescription drug costs are largely covered by each county council, which in turn receive annual reimbursement from the government. A national scheme regulates patient co-payment [7]. Patients with known $\mathrm{CHC}$ infection are mainly cared for as outpatients by hospital-based specialists in infectious diseases or gastroenterology/ hepatology. To promote efficient disease control, the cost of drugs intended for certain infectious diseases including hepatitis $\mathrm{C}$ are by the communicable disease law free of charge to the patient [8]. In a recent Swedish register-based study, the overall prevalence of $\mathrm{CHC}$ infection and treated disease are estimated to be 0.36 and $0.01 \%$, respectively [9].

Soon after the introduction of sofosbuvir and simeprevir (Fig. 1), the Medical Product Agency and their reference group of specialists on antiviral therapy published treatment guidelines based on available clinical evidence [10]. Since the new DAAs were high-priced, easier to use and
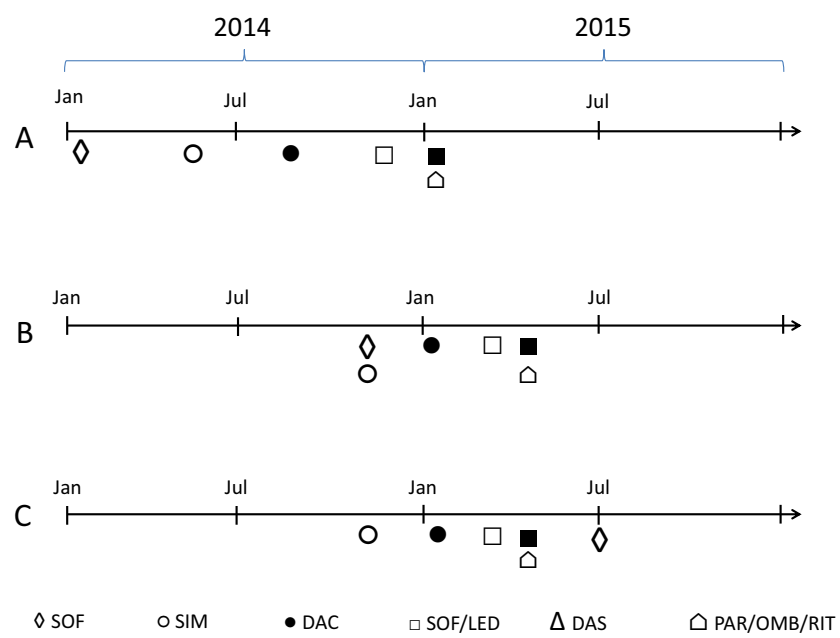

Fig. 1 Timelines for regulatory approval (A), inclusion of drug in introduction protocol (B), and risk sharing agreement $(\mathbf{C})$. SOF, sofosbuvir; SIM, simeprevir; DAC, daclatasvir; SOF/LED, sofosbuvir/ ledipasvir; DAS, dasabuvir; PAR/OMB/RIT, paritaprevir/ombitaprevir/ ritonavir very promising regarding effectiveness, a considerable increase in expenditure and potentially large regional differences in uptake was anticipated. Therefore, a national introduction process, coordinated by the Swedish Association of Local Authorities and Regions (SALAR) and involving county councils, the Medicinal Products Agency, The Dental and Pharmaceutical Benefits Agency and specialists, was initiated in early 2014 , as a pilot project guided by the new working process for the managed introduction of new drugs [11]. It resulted in an introduction protocol issued by the New Therapies Council, an expert group supporting the county councils. The protocol contained clinical background information on currently and soon to be available DAAs, and guidelines on how to prioritize patients for treatment with DAAs and on which drugs to choose. The first protocol with guidelines based on both clinical recommendations and health economic assessments was implemented on November 7th 2014, about 9 months after the regulatory approval of sofosbuvir (Fig. 1). Even though it was not mandatory from a policy perspective, the protocol was accepted by all involved actors and updated repeatedly when new drugs were launched and received a health economic assessment. Simultaneously, price negotiations between all county councils, The Dental and Pharmaceutical Benefits Agency and the pharmaceutical companies took place, resulting in risk sharing agreements. Over time, these were included in the introduction protocols since they-along with the available clinical evidence and health economic assessments - had an impact on which drugs were recommended (Table 1) [12]. Only prescribers specialized in treating patients with $\mathrm{CHC}$ were allowed to prescribe. Initially, treatment was restricted to patients with advanced fibrosis/cirrhosis (fibrosis stage 3 and 4). This restriction was relieved to also include patients with moderate fibrosis (fibrosis stage 2) from July 2nd, 2015 (Table 2). In addition, there were regional variations in other costcontainment strategies, with the southern region implementing a patient-level prior authorization scheme and the middle, northern, western, and south-east regions implementing different regional approval procedures, where clinicians could nominate new therapies for approval on a county or regional level.

Our aims were to study treatment outcome in all patients starting treatment with DAAs during 2014-2015 and to study prescribers' adherence to the drug recommendations and treatment eligibility criteria presented in the introduction protocol implemented in Sweden in 2014-2015, covering the introduction of sofosbuvir, simeprevir, daclatasvir, ledipasvir/sofosbuvir, dasabuvir, and paritaprevir/ombitasvir/ritonavir for the treatment of CHC. Furthermore, the rate of introduction in the different health care regions was studied. 
Table 1 First line treatment with DAAs by genotype (Gt), version of introduction protocol and stage of fibrosis $(\mathrm{F})$

\begin{tabular}{|c|c|c|c|c|c|}
\hline & $\begin{array}{l}2014-11-07 \\
(2.0)\end{array}$ & 2015-01-12 (3.0) & 2015-03-06 (4.0) & $2015-04-13(5.0)$ & $2015-07-02(6.0)$ \\
\hline Gt & F3-F4 & F3-F4 & F3-F4 & F3-F4 & F3-F4 \\
\hline 1 & $\mathrm{SOF}+\mathrm{SIM}$ & $\begin{array}{l}\text { Treatment naïve: } \\
\text { SOF + SIM } \\
\text { Previously treated: } \\
\text { SOF + SIM or SOF + } \\
\quad \text { DAC }\end{array}$ & $\mathrm{SOF} / \mathrm{LED}$ & $\begin{array}{l}\text { SOF/LED or OMB/PAR/RIT + } \\
\text { DAS }\end{array}$ & $\begin{array}{l}\text { SOF/LED or OMB/PAR/RIT + DAS } \\
\text { F2 } \\
\text { SOF/LED }\end{array}$ \\
\hline $\mathrm{Gt}_{2}$ & $\begin{array}{l}\text { F3-F4 } \\
\text { SOF + ribavirin }\end{array}$ & $\begin{array}{l}\text { F3-F4 } \\
\text { SOF + ribavirin }\end{array}$ & $\begin{array}{l}\mathrm{F} 3-\mathrm{F} 4 \\
\mathrm{SOF}+\text { ribavirin }\end{array}$ & $\begin{array}{l}\mathrm{F} 3-\mathrm{F} 4 \\
\mathrm{SOF}+\text { ribavirin }\end{array}$ & $\begin{array}{l}\mathrm{F} 2-\mathrm{F} 4 \\
\mathrm{SOF}+\text { ribavirin }\end{array}$ \\
\hline $\begin{array}{l}\text { Gt } \\
3\end{array}$ & F3-F4 & $\begin{array}{l}\text { F3-F4 } \\
\text { SOF + DAC }\end{array}$ & $\begin{array}{l}\text { F3-F4 } \\
\text { SOF + DAC or } \\
\quad \text { SOF/LED }\end{array}$ & $\begin{array}{l}\text { F3-F4 } \\
\text { SOF + DAC or SOF/LED }\end{array}$ & $\begin{array}{l}\text { F3-F4 } \\
\text { SOF + DAC or SOF + ribavirin } \\
\text { F2 } \\
\text { SOF + ribavirin }\end{array}$ \\
\hline Gt & F3-F4 & F3-F4 & F3-F4 & F3-F4 & F3-F4 \\
\hline 4 & $\mathrm{SOF}+\mathrm{SIM}$ & $\begin{array}{l}\text { Treatment naïve: } \\
\text { SOF + SIM } \\
\text { Previously treated: } \\
\text { SOF + SIM or SOF + } \\
\quad \text { DAC }\end{array}$ & $\mathrm{SOF} / \mathrm{LED}$ & $\mathrm{OMB} / \mathrm{PAR} / \mathrm{RIT}$ + ribavirin & $\begin{array}{l}\text { SOF/LED or OMB/PAR/RIT + } \\
\quad \text { ribavirin } \\
\text { F2 } \\
\text { SOF/LED }\end{array}$ \\
\hline
\end{tabular}

Ribavirin could be added where suitable. Treatment duration (varying with disease severity) is not presented here. Previous treatment status refers to previous use of telaprevir and boceprevir

SOF sofosbuvir, SIM simeprevir, DAC daclatasvir, SOF/LED sofosbuvir/ledipasvir, DAS dasabuvir, PAR/OMB/RIT paritaprevir/ombitasvir/ritonavir

\section{Methods}

\section{Data sources}

This was a cross-sectional study, with registry data covering the whole country. The data sources used were the Swedish Prescribed Drug Register [13] and the Swedish National Patient Register [14], both held by the National Board of Health and Welfare, and the national quality register InfCare
Hepatitis, held by the Karolinska University Hospital, Stockholm. The Swedish Prescribed Drug Register is a mandatory national register with information about each individual and their prescription drug purchases (age, sex, Anatomical Therapeutic Chemical [ATC] codes, prescribing and dispensing dates, number of packages, and prescribed dose). The National Patient Register is also a mandatory national register, with individual level data on documented main and contributory diagnoses (ICD codes) and diagnostic and

Table 2 Treatment eligibility criteria

\begin{tabular}{|c|c|c|c|}
\hline & Conditions & Data source(s) & Measurements \\
\hline \multirow[t]{2}{*}{$\begin{array}{l}\text { Treatment eligibility } \\
\text { (before July 2nd 2015) }\end{array}$} & $\begin{array}{l}\text { Stage of fibrosis 3-4 } \\
\text { and/or cirrhosis }\end{array}$ & InfCare Hepatitis & $\begin{array}{l}\text { Measured with the Batts-Ludwig or Metavir scale } \\
\text { or the corresponding disease stage measured } \\
\text { with other rating scales [27]. } \\
\text { Verified by Fibroscan measurement } \geq 9.4 \mathrm{kPa} \text {, } \\
\text { APRI-score }>2 \text {, and/or clinical diagnosis }\end{array}$ \\
\hline & $\begin{array}{l}\text { Transplantation at any } \\
\text { time before treatment } \\
\text { initiation }\end{array}$ & $\begin{array}{l}\text { National Inpatient Register } \\
\text { 1997-2015 }\end{array}$ & $\begin{array}{l}\text { ICD10-codes Z94.0 (renal transplant), Z94.1 } \\
\text { (heart transplant), Z94.2 (lung transplant), } \\
\text { Z94.3 (heart and lung transplant), and Z94.4 } \\
\text { (liver transplant) }\end{array}$ \\
\hline \multirow[t]{2}{*}{$\begin{array}{l}\text { Treatment eligibility } \\
\text { (from July 2nd 2015) }\end{array}$} & $\begin{array}{l}\text { Stage of fibrosis } 2-4 \\
\text { and/or cirrhosis }\end{array}$ & InfCare Hepatitis & $\begin{array}{l}\text { Measured with the Batts-Ludwig or Metavir scale } \\
\text { or the corresponding disease stage measured } \\
\text { with other rating scales [27]. } \\
\text { Verified by Fibroscan measurement } \geq 7.0 \mathrm{kPa} \text {, } \\
\text { APRI-score }>2 \text {, and/or clinical diagnosis }\end{array}$ \\
\hline & $\begin{array}{l}\text { Transplantation at any } \\
\text { time before treatment initiation }\end{array}$ & $\begin{array}{l}\text { National Inpatient Register } \\
\text { 1997-2015 }\end{array}$ & $\begin{array}{l}\text { ICD10 codes Z94.0 (renal transplant), Z94.1 } \\
\text { (heart transplant), Z94.2 (lung transplant), } \\
\text { Z94.3 (heart and lung transplant), and Z94.4 } \\
\text { (liver transplant) }\end{array}$ \\
\hline
\end{tabular}


clinical measures taken. It contains data on all hospitalizations and consultations to specialists in ambulatory care, but not consultations in primary care. InfCare Hepatitis is a national database including clinical data from $87 \%$ of the infectious disease clinics in Sweden, and one of the gastroenterology clinics. It was started in 2009 and serves primarily as a clinical decision support and benchmarking tool, but can also be used for research [12]. In this study, InfCare Hepatitis is considered a treatment-specific quality register, not disease specific, as the current number of untreated patients in the register is low [15].

Data from these sources were linked on an individual level, using the personal identification number (PIN) [16].

\section{Study population}

Patients who were dispensed or administered any of the following drugs in Sweden during 2014-2015 and who therefore are detected in either the Swedish Prescribed Drug Register and InfCare Hepatitis or in the Prescribed Drug Register only were included: sofosbuvir (ATC code J05AX15), simeprevir (ATC code J05AE14), daclatasvir (ATC code J05AX14), sofosbuvir/ledipasvir (ATC code J05AX65), dasabuvir (ATC code J05AX16), and ombitasvir/paritaprevir/ritonavir (ATC code J05AX67).

\section{Definitions}

The analysis of treatment outcome was based on total treatment given, including potential add-on treatment given when the patient did not respond sufficiently to the initial treatment and re-treatment for patients with a relapse.

Adherence to drug recommendations was defined as initiation of treatment with the drug or drugs recommended for the genotype in question at the time of treatment initiation. This analysis includes all patients with genotype 1-4 starting treatment from November 7th 2014, when the introduction protocol was implemented. Time of treatment initiation is based on the date of first dispensing in the Prescribed Drug Register or the treatment start date in InfCare Hepatitis (for patients without data in the Prescribed Drug Register).

The treatment eligibility criteria before and after July 2nd, 2015 respectively, were based on stage of fibrosis, prevalence of cirrhosis, and/or previous transplantation (Table 2). Adherence to the treatment eligibility criteria was defined as initiation of treatment in a patient who, at the time of initiation, did fulfill at least one of the criteria.

\section{Outcome measures}

Treatment outcome, i.e. cure rate, was measured as the proportion of patients per genotype who achieved a sustained viral response, i.e. undetectable levels of HCV-RNA in plasma 12 weeks after end of treatment (SVR12).

Adherence to the introduction protocol was measured as follows:

a) the proportion of patients who were treated with the drug or drugs recommended as first-line treatment for their specific genotype at the time of treatment initiation and

b) the proportion of patients who met the treatment eligibility criteria before and after July 2nd, 2015, at the time of treatment initiation.

Introduction rate was measured as the relative proportion of patients on treatment per region over time, and comparisons were made between the six healthcare regions: northern, middle, south-east, western and southern Sweden, and the region of Stockholm-Gotland, respectively. The general population distribution of the regions was based on population statistics dated June 30th 2015, from Statistics Sweden.

InfCare completeness was calculated as the number of patients with the specific characteristic in InfCare divided by the number of patients in the study cohort.

\section{Statistics}

Descriptive statistics, such as numbers and proportions, were used to describe the study cohorts and the utilization patterns, with $95 \%$ confidence intervals $(95 \% \mathrm{CI})$ where appropriate. Analyses were stratified by sex and age in the categories 0-20, 21-30, 31-40, 41-50, 51-60, 61-70, 71-80 and $\geq 81$ years. Means were presented with standard deviations (SD). Data were analyzed in SAS Enterprise Guide 6.1. (SAS Institute, Cary, NC).

\section{Ethics}

The study was reviewed and approved by the Regional Ethical Review Board in Stockholm, approval no. 2015/497-31/1.

\section{Results}

The cohort of patients initiated on the drugs included consisted of 3447 patients. In a majority of patients, clinical data relevant for the aims of this study were available (Table 3).

The analysis of treatment outcome included all patients with documented viral load measurements 12 weeks after the end of treatment (2921 patients, $85 \%$ of the cohort). Sustained viral response was achieved in $96.1 \%$ of the patients, with some variation between genotypes (Table 4).

The analysis of adherence to drug recommendations includes all patients with genotype 1-4 treated from 
Table 3 Characteristics of patient population

\begin{tabular}{lll}
\hline No. of patients & 3447 & $\%$ of cohort with missing data \\
\hline Age (years), mean (SD) & $55.8(10.8)$ & 0 \\
$0-20$ & $10(0.3 \%)$ & \\
$21-30$ & $95(2.8 \%)$ \\
$31-40$ & $250(7.3 \%)$ \\
$41-50$ & $544(15.8 \%)$ \\
$51-60$ & $1404(40.7 \%)$ \\
$61-70$ & $957(27.8 \%)$ \\
$71-80$ & $179(5.2 \%)$ \\
$81-$ & $8(0.2 \%)$ \\
Proportion women & $33.5 \%$ \\
Genotype $(n=3016)$ & \\
1 & $1708(56.6 \%)$ \\
2 & $317(10.5 \%)$ \\
3 & $870(28.8 \%)$ \\
4 & $112(3.7 \%)$ \\
5 & $2(0.1 \%)$ \\
6 & $7(0.2 \%)$ \\
Estimated stage of fibrosis at treatment initiation* $(n=2672)$ & \\
F0-F1 & $319(11.9 \%)$ \\
F2 & $473(17.7 \%)$ \\
F3 & $588(22.0 \%)$ \\
F4 & $1292(48.4 \%)$ \\
Previous transplantation & $266(7.7 \%)$ \\
\hline
\end{tabular}

* Registered stage of fibrosis in InfCare Hepatitis is often based on liver biopsy. Where a registered stage of fibrosis was missing; an estimated value was assigned based on measurement of liver elasticity (Fibroscan) or APRI-score, if possible
November 7th 2014 (2253 patients, 65\% of the cohort). On an overall level, adherence to drug recommendations was initially moderate, $43.2 \%$ (95\% CI 37.5-48.9\%), and increased over time to $94.2 \%$ (95\% CI $92.7-95.6 \%$ ), for treatment initiation done after July 2nd 2015. Adherence varied considerably between genotypes (Table 5).

The analysis of treatment eligibility criteria includes all patients with at least one marker for disease stage (2672 patients, 78\% of the cohort). Before and after July 2nd 2015,

Table 4 Cure rates per genotype

\begin{tabular}{lrl}
\hline Genotype & No. of patients & $\begin{array}{l}\text { Proportion with } \\
\text { SVR12 }( \pm 95 \% \mathrm{CI})\end{array}$ \\
\hline 1 & 1631 & $97.6 \pm 0.8$ \\
2 & 289 & $95.2 \pm 2.5$ \\
3 & 788 & $93.8 \pm 1.7$ \\
4 & 107 & $97.2 \pm 3.1$ \\
5 & 2 & 100.0 \\
6 & 7 & 100.0 \\
Genotype missing & 97 & $92.8 \pm 5.2$ \\
Total & 2921 & $96.1 \pm 0.7$ \\
\hline
\end{tabular}

adherence to the treatment eligibility criteria was 80 and $87 \%$ respectively, with minor differences in adherence rates between the health care regions (Figs. 2 and 3).

Although the first protocol was implemented in November 2014 (Fig. 1), prescribing to patients started as soon as the first drugs were available. However, the rate of introduction varied initially between regions, and approached stable levels 15 months after the introduction of the drugs started (Fig. 4).

\section{Discussion}

This cross-sectional study with national data on all patients selected for treatment with DAAs during 2014-2015 reflects the use of these drugs their first 2 years on the market, and covers a majority of the population initiated on treatment in Sweden during those years. It is not intended as a direct comparison between individual drugs, but to describe the patients treated and measure the extent to which the recommendations in the introduction protocol were implemented. Cure rates, based on data from $85 \%$ of the cohort, were generally as high as expected from either RCTs [17] or recent observational studies [18-23], with some variation between genotypes. 
Table 5 Adherence to drug recommendations over time

\begin{tabular}{|c|c|c|c|c|c|c|}
\hline \multirow[t]{2}{*}{ Genotype } & \multicolumn{5}{|c|}{ Protocol version (proportion with prescribing as recommended, $\pm 95 \% \mathrm{CI}$ ) } & \multirow[b]{2}{*}{$n$} \\
\hline & 2.0 & 3.0 & 4.0 & 5.0 & 6.0 & \\
\hline 1 & $57.6 \pm 7.6 \%$ & $79.8 \pm 5.6 \%$ & $72.7 \pm 7.4 \%$ & $94.0 \pm 3.2 \%$ & $94.3 \pm 1.9 \%$ & 1302 \\
\hline 2 & $92.6 \pm 9.9 \%$ & $85.4 \pm 10.9 \%$ & $84.2 \pm 16.4 \%$ & $92.3 \pm 10.3 \%$ & $82.8 \pm 7.5 \%$ & 212 \\
\hline 3 & $0 \%$ & $91.7 \pm 5.0 \%$ & $96.7 \pm 4.5 \%$ & $96.3 \pm 3.6 \%$ & $97.9 \pm 3.4 \%$ & 663 \\
\hline 4 & $66.7 \pm 37.8 \%$ & $88.9 \pm 14.5 \%$ & $85.7 \pm 26.4 \%$ & $15.4 \pm 19.6 \%$ & $93.8 \pm 8.4 \%$ & 76 \\
\hline
\end{tabular}

Includes all patients with documented genotype 1-4, starting treatment as of November 7th, 2014 (2253 patients, $65 \%$ of population)
Most of the patients were treated with the recommended combinations of the drugs under study, and the initially moderate adherence to the drug recommendations increased over time, with the increasing inclusion of drugs and drug combinations in the introduction protocol. Adherence to the treatment eligibility criteria was high and increased further when the restrictions were widened to also include patients with moderate liver disease. The rate of introduction varied between the regions, ranging between the more rapid uptake seen in the region of Stockholm-Gotland and the somewhat slower uptake seen in the western region.

The uptake of drugs among specialists is influenced by several factors [24] and other recent introductions in Sweden were less uniform and rapid, despite the implementation of a national introduction protocol [25]. The faster uptake of the DAAs in the region of Stockholm-Gotland, with approximately $25-30 \%$ of the Swedish CHC-population, is likely attributable to a larger number of prescribers with experience of using the drugs being introduced in earlier and ongoing clinical trials, and the fact that this region did not implement any further cost containment strategies than the introduction protocol. A recently published governmental investigation of the introduction process concluded that despite some initial regional variations in uptake of $\mathrm{CHC}$ treatment, the national

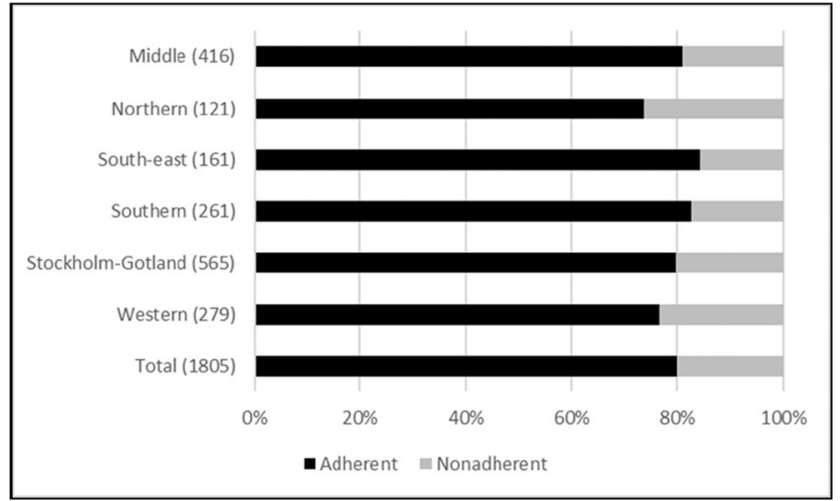

Fig. 2 Prescribers' adherence to treatment eligibility criteria effective before July 2nd 2015, presented by healthcare region. Data representing two patients with missing information on county of residence are omitted from the region columns, but included in total. The number of patients per region is presented within brackets introduction could be considered as uniform over the country [25].

Considering also the regional variations in other cost containment strategies and differences in tax base and patient populations between counties and regions, the results of this study demonstrate a considerable loyalty to the introduction process and protocol. The rate of adherence to treatment eligibility criteria was generally high, reflecting a high awareness among prescribers regarding the considerable costs and the need to restrict prescribing to those with the more severe stages of disease, even before the introduction protocols were implemented. Non-adherence may be related to occasional prescribing for unaccounted medical reasons or regional variations in disease severity among patients; this can however not be detected in the database. The initially moderate adherence to drug recommendations reflects the restricted number of drugs included in the protocol compared to the number of substances available on the market, but potentially also an initial variation in knowledge of the protocol itself. The legal status of the recommendations in the national introduction protocols - in relation to the existing legal framework - may also have been unclear to prescribers. To further legitimize the national introduction process and improve adherence to the future introduction protocols, this needs to be clarified [25].

Strengths of this study include a database with complete coverage on all patients' prescribed/dispensed DAAs and

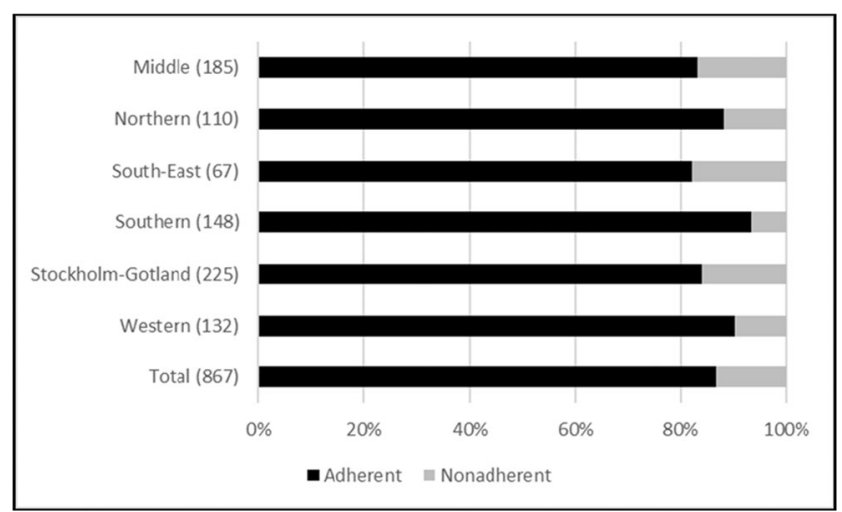

Fig. 3 Prescribers' adherence to treatment eligibility criteria effective from July 2nd 2015, presented by healthcare region. The number of patients per region is presented within brackets 


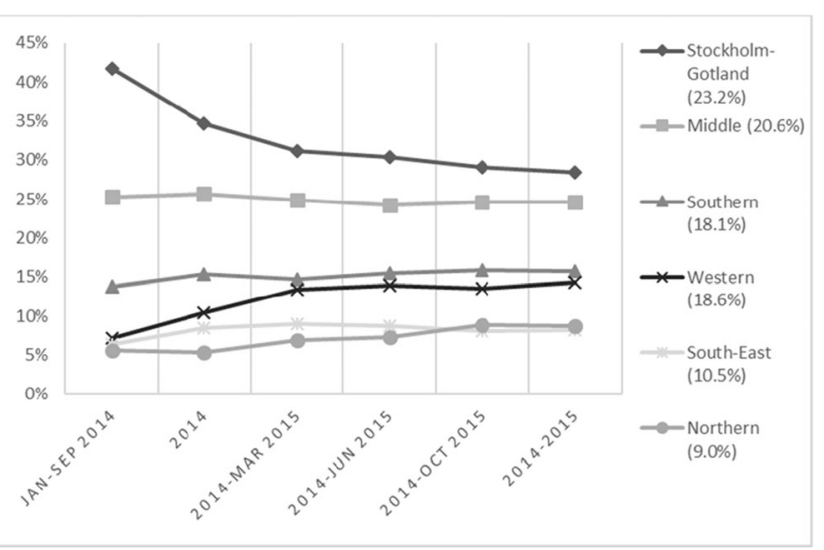

Fig. 4 Distribution of treated patients by health care region over time. Percentages within brackets represent each region's proportion of the general population as of June 30th 2015

clinical data on genotypes; stage of fibrosis and outcomes for almost $90 \%$ of the treated patients making is possible to analyze both drug utilization patterns and treatment outcomes. However, the study has several limitations. Even though the risk sharing agreements and the targeted governmental funding are considered important factors for this particular introduction [25], this study was not designed to determine the relative impact of the different, simultaneously implemented and rapidly changing policy measures on prescribing and uptake of the DAAs. Nor is it designed to detect and compare rates of undertreatment over time, i.e. the extent to which patients eligible for treatment based on disease severity are left without treatment. This would indeed have given a broader picture of the introduction rate than merely monitoring the relative proportion of patients initiated on treatment, but was not possible due to the limited amount of clinical data on untreated patients during 2014-2015. The definition of treatment eligibility is based on what can be reliably captured in either of the national registers used. Geographically varying completeness in InfCare Hepatitis and access to Fibroscan equipment for measurement of liver elasticity may have caused some patients to be misclassified as non-eligible, since their disease stage was not sufficiently documented or documented, but based on older liver biopsies. From July 2015, women with $\mathrm{CHC}$ preparing for in vitro fertilization were also considered eligible for treatment; this could however not be reliably captured and may thus have contributed to an underestimation of adherence to the treatment eligibility criteria. Patients treated as inpatients in hospitals were only included in the cohort if they were either registered in InfCare Hepatitis or had part of their drugs dispensed as prescription drugs. This may have influenced the patient population selected for this study as well as the estimated coverage of InfCare Hepatitis. However, the total drug volume of all patients completely or partially treated as inpatients, measured as packages sold over 2014-2015, is less than $2 \%$ of total volume [26]; hence, the impact of this methodological problem is likely to be minor.

\section{Conclusion}

The conditions for introducing the first six of the secondgeneration direct-acting antivirals for chronic hepatitis $\mathrm{C}$ infection in Sweden were outlined in a national introduction protocol. The overall cure rate was estimated to $96 \%$, with some variation between genotypes. Despite regional variation in other cost containment strategies, the high level of adherence to recommendations among prescribers and similar introduction rates in the regions indicate that the protocol contributed considerably to a rapid uptake and equal distribution of DAAs in Sweden.

Acknowledgements We gratefully acknowledge Anna Bennet Bark at the National Board of Health and Welfare, Pontus Johansson at the Dental and Pharmaceutical Benefits Agency and Olle Karlström at the Medical Products Agency for their valuable input to the study design.

Contributions of authors PF contributed to the design of study, acquisition, analysis and interpretation of data, and produced the first and subsequent drafts. KA and SL contributed to the design of study and critically revised the drafts. TC contributed to the acquisition and analysis of data, and critically revised the drafts. NF contributed to the design of study and critically revised the drafts. BW contributed to the design of the study and interpretation of data, and critically revised the drafts. OW contributed to the design of the study, acquisition and interpretation of data, and critically revised the drafts. All authors read and approved the final manuscript.

Funding This study was jointly funded by all Swedish county councils.

\section{Compliance with ethical standards}

Conflict of interest The authors declare that they have no conflicts of interest.

Disclosure statement The views in this paper are those of the authors and not necessarily representative of the governmental agencies at which they are employed.

Open Access This article is distributed under the terms of the Creative Commons Attribution 4.0 International License (http:// creativecommons.org/licenses/by/4.0/), which permits unrestricted use, distribution, and reproduction in any medium, provided you give appropriate credit to the original author(s) and the source, provide a link to the Creative Commons license, and indicate if changes were made.

\section{References}

1. Wang LS, D'Souza LS, Jacobson IM (2016) Hepatitis C — a clinical review. J Med Virol 88(11):1844-1855. https://doi.org/10.1002/ jmv. 24554

2. Mohd Hanafiah K, Groeger J, Flaxman AD, Wiersma ST (2013) Global epidemiology of hepatitis $\mathrm{C}$ virus infection: new estimates of age-specific antibody to HCV seroprevalence. Hepatology 57(4): 1333-1342

3. Chou R, Hartung D, Rahman B, Wasson N, Cottrell EB, Fu R (2013) Comparative effectiveness of antiviral treatment for hepatitis 
$\mathrm{C}$ virus infection in adults: a systematic review. Ann Intern Med 158(2):114-123

4. Liang TJ, Ghany MG (2013) Current and future therapies for hepatitis C virus infection. N Engl J Med 368(20):1907-1917. https:// doi.org/10.1056/NEjMra1213651

5. Brennan T, Shrank W (2014) New expensive treatments for hepatitis C infection. JAMA 312(6):593-594

6. Anell A, Glenngård AH, Merkur S (2012) Sweden health system review. Health Syst Transit 14(5):1-159

7. TLV. Patient co-payment scheme for prescription drugs. [Internet]. Accessed 5 October 2017. http://www.tlv.se/In-English/medicinesnew/the-swedish-high-cost-threshold/how-it-works/

8. SFS 2004:168 Swedish government Communicable disease law [Internet]. Accessed 5 October 2017. http://www.riksdagen.se/sv/ dokument-lagar/dokument/svensk-forfattningssamling/ smittskyddslag-2004168_sfs-2004-168

9. Büsch K, Waldenström J, Lagging M, Aleman S, Weiland O, Kövamees J, Duberg AS, Söderholm J (2016) Prevalence and comorbidities of chronic hepatitis C: a nationwide population-based register study in Sweden. Scand J Gastroenterol 52:61-68. https:// doi.org/10.1080/00365521.2016.1228119

10. Läkemedelsbehandling av hepatit $\mathrm{C}$-virusinfektion hos vuxna och barn - kunskapsunderlag. Information från Läkemedelsverket. 2014.

11. Sveriges kommuner och landsting (2014) Ordnat införande i samverkan: slutrapport från nationella läkemedelsstrategin, delprojekt 6.1. Sveriges Kommuner och Landsting, Stockholm

12. New drug therapies for hepatitis $\mathrm{C}$ A national introduction- and monitoring protocol (version 6.0) [Internet]. Accessed 18 August $2016 \mathrm{http} / / / \mathrm{www} . j a n u s i n f o . s e / D o c u m e n t s / N a t i o n e l l t \_i n f o r a n d e$ av nya lakemedel/Inforande-och-uppfoljningsprotokoll-forlakemedel-mot-hepatit-C-Version-6.pdf

13. Wettermark B, Hammar N, Fored CM, MichaelFored C, Leimanis A, Otterblad Olausson P et al (2007) The new Swedish Prescribed Drug Register-opportunities for pharmacoepidemiological research and experience from the first six months. Pharmacoepidemiol Drug Saf 16(7):726-735

14. Ludvigsson JF, Andersson E, Ekbom A, Feychting M, Kim J-L, Reuterwall C, Heurgren M, Olausson PO (2011) External review and validation of the Swedish national inpatient register. BMC Public Health 11:450. https://doi.org/10.1186/1471-2458-11-450.

15. Emilsson L, Lindahl B, Köster M, Lambe M, Ludvigsson JF (2015) Review of 103 Swedish healthcare quality registries. J Intern Med 277(1):94-136

16. Ludvigsson JF, Otterblad-Olausson P, Pettersson BU, Ekbom A (2009) The Swedish personal identity number: possibilities and pitfalls in healthcare and medical research. Eur J Epidemiol 24(11):659-667

17. Ahmed H, Abushouk AI, Menshawy A, Mohamed A, Negida A, Loutfy SA, Abdel-Daim MM (2017) Safety and efficacy of ombitasvir/paritaprevir/ritonavir and dasabuvir with or without ribavirin for treatment of hepatitis $\mathrm{C}$ virus genotype 1: a systematic review and meta-analysis. Clin Drug Investig 37:1009-1023. https://doi.org/10.1007/s40261-017-0565-5. [Epub ahead of print]

18. Flisiak R, Pogorzelska J, Flisiak-Jackiewicz M (2017) Hepatitis C: efficacy and safety in real life. Liver Int 37(Suppl 1):26-32. https:// doi.org/10.1111/liv.13293

19. Welzel TM, Petersen J, Herzer K, Ferenci P, Gschwantler M, Wedemeyer H, Berg T, Spengler U, Weiland O, van der Valk M, Rockstroh J, Peck-Radosavljevic M, Zhao Y, Jimenez-Exposito MJ, Zeuzem S (2016) Daclatasvir plus sofosbuvir, with or without ribavirin, achieved high sustained virological response rates in patients with HCV infection and advanced liver disease in a realworld cohort. Gut 65(11):1861-1870

20. Maria C, Michael S, Susanne C, Catarina S, Ola W (2017) INF-free sofosbuvir-based treatment of post-transplant hepatitis C relapse- a Swedish real life experience. Scand J Gastroenterol 52(5):585-588

21. Dalgard O, Weiland O, Noraberg G, Karlsen L, Heggelund L, Färkkilâ M, Balslev U, Belard E, Øvrehus A, Skalshøi Kjær M, Krarup H, Thorup Røge B, Hallager S, Madsen LG, Lund Laursen A, Lagging M, Weis N (2017) Sofosbuvir based treatment of chronic hepatitis $\mathrm{C}$ genotype 3 infections - a Scandinavian real-life study. PLoS One 12(7):e0179764

22. Young J, Weis N, Hofer H, Irving W, Weiland O, Giostra E, Pascasio JM, Castells L, Prieto M, Postema R, Lefevre C, Evans D, Bucher HC, Calleja JL (2017) The effectiveness of daclatasvir based therapy in European patients with chronic hepatitis $\mathrm{C}$ and advanced liver disease. BMC Infect Dis 17(1):45. https://doi.org/ 10.1186/s12879-016-2106-x

23. Wehmeyer MH, Ingiliz P, Christensen S, Hueppe D, Lutz T, Simon KG, Schewe K, Boesecke C, Baumgarten A, Busch H, Rockstroh J, Schmutz G, Kimhofer T, Berger F, Mauss S, Schulze zur Wiesch J (2017) Real-world effectiveness of sofosbuvir-based treatment regimens for chronic hepatitis $\mathrm{C}$ genotype 3 infection: results from the multicenter German hepatitis C cohort (GECCO-03). J Med Virol 90:304-312. https://doi.org/10.1002/jmv.24903. [Epub ahead of print]

24. Chauhan D, Mason A (2008) Factors affecting the uptake of new medicines in secondary care a literature review. J Clin Pharm Ther 33(4):339-348

25. Ordning i leden? Utvärdering av ordnat införande av nya läkemedel. Rapport 2017:5 [Internet]. Myndigheten för vård- och omsorgsanalys. Accessed 15 August 2017. www.vardanalys.se/ Rapporter/2017/Ordning-i-leden/

26. Aggregated statistics from the Swedish eHealth Agency [Internet]. www.ehalsomyndigheten.se

27. Goodman ZD (2007) Grading and staging systems for inflammation and fibrosis in chronic liver diseases. J Hepatol 47(4):598-607 